\title{
Symptom variability in COPD: a narrative review
}

This article was published in the following Dove Press journal:

International Journal of COPD

6 May 2013

Number of times this article has been viewed

\author{
Jose Luis Lopez-Campos ${ }^{1,2}$ \\ Carmen Calero ${ }^{1,2}$ \\ Esther Quintana-Gallego ${ }^{1,2}$ \\ 'Unidad Medico-Quirúrgica de \\ Enfermedades Respiratorias, \\ Instituto de Biomedicina de Sevilla \\ (IBiS), Hospital Universitario Virgen \\ del Rocio, Seville, Spain; ${ }^{2}$ CIBER \\ de Enfermedades Respiratorias \\ (CIBERES), Instituto de Salud \\ Carlos III, Madrid, Spain
}

\begin{abstract}
Chronic obstructive pulmonary disease (COPD) has traditionally been considered an inexorably progressive disease, associated with a constant increase of symptoms that occur as the forced expiratory volume in 1 second $\left(\mathrm{FEV}_{1}\right)$ worsens, only intermittently interrupted by exacerbations. However, this paradigm has been challenged in recent decades by the available evidence. Recent studies have pointed out that COPD-related symptoms are not consistently perceived by patients in the same way, showing not only seasonal variation, but also changes in symptom perception during a week or even within a single day. According to the available data, patients experience the biggest increase in respiratory symptoms during the first hours of the early morning, followed by the nighttime. This variation over time is of considerable importance, since it impacts on daily life activities and health-related quality of life, as measured by a recently developed ad hoc questionnaire. Additionally, recent clinical trials have suggested that the use of rapid-onset long-acting bronchodilators may have an impact on morning symptoms, despite their current use as maintenance treatment for a determined period. Although this hypothesis is to be validated in future long-term clinical trials comparing fast-onset versus slow-onset inhaled drugs in COPD, it may bring forward a new concept of long-term bronchodilator therapy. At the present time, the two available long-acting, fast-onset bronchodilators used in the treatment of COPD are formoterol and the recently marketed indacaterol. Newer drugs have also been shown to have a rapid onset of action in preclinical studies. Health care professionals caring for COPD patients should consider this variation in the perception of symptoms during their clinical interview as a potential new target in the long-term treatment plan.
\end{abstract}

Keywords: symptom perception, rapid-onset bronchodilators, indacaterol, formoterol, glycopyrronium, aclidinium

\section{Introduction}

Despite their enormous heterogeneity, chronic airway diseases share various relevant aspects of clinical presentation. From the functional standpoint, the impaired ability to exhale inspired air is characteristic of this group of diseases, which cause alterations in ventilatory mechanics of varying degrees. From a biological standpoint, the presence of an inflammation of the bronchial wall has turned out to be a constant feature, although this may differ in the nature or intensity. Finally, and as a result of the foregoing, the increased perception of symptoms has a key impact on health-related quality of life (HRQL), affecting activities of daily living. In this regard, the perception that patients have about their chronic respiratory symptoms is a relevant measure of the impact of their disease, which directly affects their functional capacity, physical activity, HRQL, and prognosis. ${ }^{1}$ This is of particular importance in patients with chronic obstructive
Correspondence: Jose Luis Lopez-Campos Hospital Universitario Virgen del Rocio, Avda Manuel Siurot, s/n, 41013, Seville, Spain

Tel +34955013167

Fax +3495 5013167

Email Icampos@separ.es 
pulmonary disease (COPD), due to the progressive nature of this disease, traditionally associated with a progressive increase of symptoms together with respiratory and systemic complications that occur as the forced expiratory volume in 1 second $\left(\mathrm{FEV}_{1}\right)$ worsens (Figure 1$)$. Further, it is frequently stated that COPD is a chronic condition with persistent symptoms that remain unchanged, only intermittently interrupted by exacerbations. ${ }^{2,3}$

This paradigm, which has accompanied the disease for decades, has been recently challenged by the available evidence. Firstly, not all patients with COPD demonstrate deterioration in $\mathrm{FEV}_{1}$ in a similar manner. In a recent analysis of the Evaluation of COPD Longitudinally to Identify Predictive Surrogate Endpoints (ECLIPSE) study, Vestbo et al evaluated the changes in $\mathrm{FEV}_{1}$ of the COPD cohort over 3 years of follow-up. ${ }^{4}$ The analysis showed that there were large variations among patients in the decline in $\mathrm{FEV}_{1}$. Interestingly, some patients gained lung function over the years of study, challenging the idea that it is not possible to recover from the airway obstruction. ${ }^{5}$ These findings were replicated in a different cohort. ${ }^{6}$ Secondly, not all symptoms increase in a similar manner with worsening $\mathrm{FEV}_{1}$, as found by the ECLIPSE study; ${ }^{7}$ while it is true that patients have more symptoms as $\mathrm{FEV}_{1}$ decreases, it is also true that there are many patients with few symptoms at all levels of disease severity. ${ }^{7}$ Therefore, there is a poor correlation between symptom perception and $\mathrm{FEV}_{1}$. It follows that the assessment of the disease should be multidimensional such that several aspects of the clinical presentation of the disease, such as symptom perception, limitation in physical activity, lung function, and exacerbations, interrelate to configure the final disease expression. ${ }^{8}$ In this new conception of COPD, the correct evaluation of symptoms and their variability is a key component for ensuring an adequate treatment strategy. ${ }^{9}$ In the following paragraphs we will review the available evidence on the variability of clinical symptoms in COPD.

\section{Symptom variability exists in COPD}

Traditionally, COPD has been considered a relentlessly progressive disease, in which the deterioration in lung function was associated with an increase in symptoms (Figure 1), interrupted only by the appearance of exacerbations. ${ }^{2,3}$ Nonetheless, recent studies suggest the opposite, indicating that the perception of symptoms may not be as stable as previously suggested. One study conducted Internet interviews with 803 COPD patients from Europe and USA, including 289 patients with severe COPD, in regard to regular use of COPD medication, chronic symptoms, and the occurrence of exacerbations. ${ }^{10}$ The study highlighted, for the first time, that morning was the worst time of day, particularly in patients with severe COPD, as reported by $46 \%$ of these patients. Shortness of breath was the most frequently reported symptom, and this perception correlated strongly with the limitations experienced during the morning activities. Interestingly, the majority of patients did not adjust their medication in time to control these symptoms. The study also identified nighttime as important in terms of symptom perception. Interestingly, patients experiencing

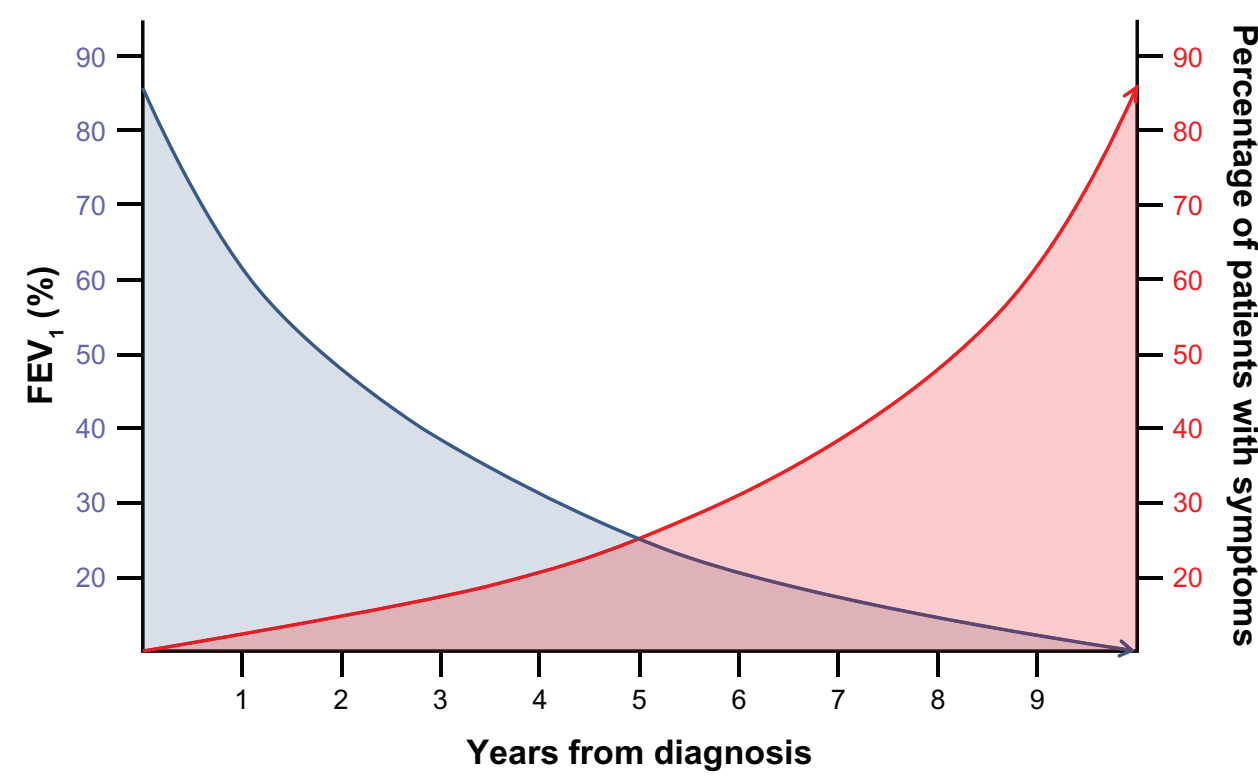

Figure I Conventional conception of the relationship between lung function and symptoms in COPD. Abbreviations: COPD, chronic obstructive pulmonary disease; $\mathrm{FEV}_{\mathrm{l}}$, forced expiratory volume in I second. 
general fatigue and tiredness were more likely to experience nighttime symptoms, suggesting an impaired sleep quality as a consequence of nighttime COPD symptoms. In this regard, an unexpected association between chronic back pain and nighttime COPD symptoms was observed, indicating that those patients with sleep disturbances due to back pain may be more aware of their COPD symptoms. ${ }^{10}$

In a pan-European cross-sectional observational study, a telephone survey was conducted in 17 countries that aimed to evaluate COPD symptom variability in daily life. ${ }^{11}$ This study assessed symptom variability in 2441 patients during a 7-day period. The vast majority of the studied patients (92.5\%) experienced at least one symptom related to COPD, including breathlessness, phlegm, cough, wheezing, or chest tightness. Of these, dyspnea was, by far, the most commonly reported (72.5\%). However, these symptoms were not perceived as constant, and $62.7 \%$ perceived variation in one or more of these symptoms. This variability was perceived all year long by $59.5 \%$, worsening during the winter months. This seasonal variation had also been described previously, and manifested in a higher number of exacerbations during cold months. ${ }^{12}$ However, one outstanding observation was that $54.4 \%$ of study patients experienced variability in their perception of symptom throughout the week. Additionally, $44.7 \%$ perceived this variability even within the same day. Altogether, daily or weekly symptom variability was reported by $62.7 \%$ of symptomatic patients. Interestingly, chest tightness and dyspnea were the most variable symptom over the week and during a single day, followed by wheezing.

The question therefore arises as to what time of the day is more profoundly impaired by these variably perceived symptoms. Since symptom perception is well known to be correlated with functional status and HRQL, ${ }^{13}$ it was expected that those hours of most intense activity would be more profoundly impaired and symptoms perceived as worse. However, generally, this seemed not to be the case. According to the study by Kessler et al, ${ }^{11}$ symptom distribution was more importantly perceived in the first hours of the morning (Figure 2). Altogether, the percentage of patients who reported troublesome symptoms upon waking and later in the morning was $45.4 \%$ for breathlessness, $60.1 \%$ for cough, $70.9 \%$ for phlegm, $45.4 \%$ for chest tightness, and $43.4 \%$ for wheezing. Nighttime was identified as another troublesome period for a number of patients who reported wheeze $(25.1 \%)$, cough $(17.3 \%)$, chest tightness $(16.7 \%)$, phlegm (11.8\%), or breathlessness (10.6\%). Consequently, $26.5 \%$ of patients reported that their symptoms affected sleep quality. ${ }^{11}$

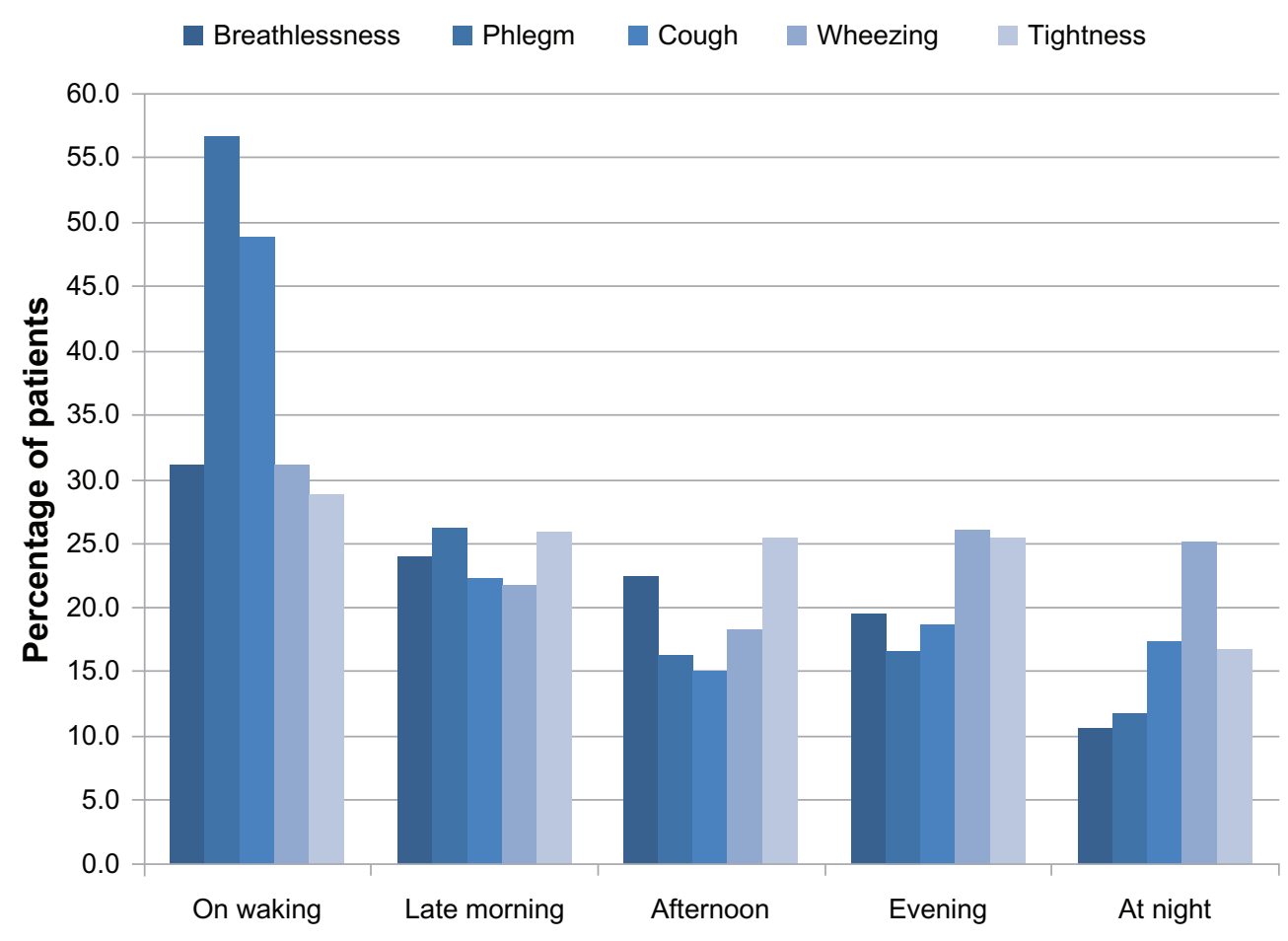

Figure 2 Symptom perception during the day. 
In the Spanish branch of this European study, ${ }^{11}$ Espinosa de los Monteros et $\mathrm{al}^{14}$ evaluated 472 patients and found that the most frequent symptom was expectoration $(55.9 \%)$, followed by dyspnea (49.8\%). Most of the patients also reported perceiving variation in symptoms during the year, the week, or within the same day. In this regard, $69.2 \%$ reported that their symptoms varied in accordance with the seasons of the year, with worsening symptoms in winter and in summer, and $60.9 \%$ declared that at least one symptom varied over the course of the day or the week. In these patients, there was a clear predominance of morning symptoms, especially for cough and sputum production. In this substudy, around $30 \%$ of the patients reported some degree of impairment in their morning activities, such as getting up, washing, or dressing. Interestingly, the second most important period of impairment was the night, with an increase in respiratory symptoms, such as wheezing or chest tightness, and sleep quality was disturbed in $19 \%$ of this population.

Finally, in a recent Korean study, Kim et al prospectively recorded data on the symptom perception of 133 COPD patients with severe airflow limitation. ${ }^{15}$ The authors found that $57 \%$ of patient-reported morning symptoms. Additionally, COPD-related symptoms were more frequent and severe in patients with morning symptoms. These were less frequently treated with a fixed-dose combination of a long-acting $\beta_{2}$ agonist (LABA) and an inhaled corticosteroid. Interestingly, there was no correlation between the severity of the obstruction and the severity of the impairment for any of the morning activities.

All of the abovementioned studies focused on respiratory symptoms alone. Nevertheless, COPD has systemic implications and is often associated with comorbidities that contribute to the overall severity of the disease. ${ }^{16}$ In turn, these systemic manifestations express their own symptoms, which may also prove variable in time. Unfortunately, the variability of symptoms related to systemic COPD manifestations or comorbidities has not been assessed so far and constitutes an interesting area for research.

\section{Importance of symptom variability}

The importance of the variability of symptoms in COPD has been verified in the context of different temporal periods. There are several previous works assessing seasonal variability that indicate an impact not only on the frequency of exacerbations, but also in their severity. ${ }^{12,17}$ There is also evidence evaluating the impact of symptom variability during the week. Another study evaluated the importance of the variation of symptom perception during the week, assessing the probability of the patient being admitted to the hospital during the weekends and the related clinical outcomes. ${ }^{18}$ The authors observed an increase in hospital mortality for those patients admitted during the weekends. However, the impact of perceived symptom variability during the hours of the same day has been scarcely studied.

In the study by Kessler et al, ${ }^{11}$ morning activities were described to be very severely impaired in COPD. Accordingly, the morning activities that patients felt were most affected by their COPD symptoms were washing, dressing, drying after bathing, and getting out of bed. In total, 9.5\% of patients reported that they required assistance in performing their usual morning activities and that they worried about being a burden to their relatives. Interestingly, the impact on activities was increased in those patients who reported the greatest variability of symptoms compared with those who had minimal variation. In the Spanish branch of the study, ${ }^{14}$ the most affected morning activities were getting out of bed, personal hygiene, and getting dressed. Additionally, $19 \%$ declared that their respiratory symptoms affected their sleep quality.

The importance of this variability in symptoms can be evaluated with respect to the different degrees of disease severity. In this regard, it is well known that patients in advanced stages of the disease experience more severe symptoms compared with those in milder stages of the disease. ${ }^{7}$ However, only a few studies have evaluated this hypothesis with respect to the experience of morning symptoms in COPD. In one such study, which involved the development of a specific questionnaire for morning symptoms, ${ }^{19}$ the authors did not find relevant differences in the perception of morning symptoms according to the Global Initiative for Chronic Obstructive Lung Disease (GOLD) staging system, suggesting that these symptoms are important in all COPD patients, regardless of the disease stage as measured by $\mathrm{FEV}_{1}$. Interestingly, nighttime symptoms have been described to be related to the lung function impairment, increasing the prevalence of these symptoms as the $\mathrm{FEV}_{1}$ deteriorates. ${ }^{20}$ This discrepancy opens an interesting debate about the impact of this recently observed phenomenon on COPD and constitutes a call for research in this area. Additionally, a potential link between early morning and nighttime symptoms in COPD is also unclear and deserves further research.

An interesting clinical association between symptom variability and exacerbation was recently established using the data from the COPD and Seretide: a Multi-Center Intervention and Characterization (COSMIC) Study, which 
investigated the effects of the withdrawal of fluticasone in patients with COPD. ${ }^{21}$ In this analysis, the authors evaluated the symptom diary cards of 373 COPD patients and evaluated symptom progression during the days previous to and after 492 exacerbations. ${ }^{22}$ The perception of cough, sputum, dyspnea, and nocturnal awakenings steadily increased beginning 2 weeks prior to an exacerbation. Interestingly, symptom variability did not change prior to the first and second moderate exacerbation. However, symptom variability was rather considerable in the days before a hospital admission, suggesting that daytime and nighttime symptom variability may predict a new severe exacerbation. This finding is in line with that of the study by Kessler et al, ${ }^{11}$ in which patients who had experienced frequent exacerbations in the previous 12 months were more likely to experience variability in breathlessness over the course of a week.

The assessment of the impact of symptom variability on HRQL was not possible until the very recent development of specific questionnaires. Partridge et al developed one such questionnaire (discussed in the following section) to assess symptom perceptions during the morning, in COPD patients. ${ }^{19}$ According to this study, ${ }^{19}$ morning symptoms seem to have an important impact on daily living, suggesting that the type of treatment used may influence symptom perception in the morning and thus improve HRQL. ${ }^{23}$ Studies measuring the impact of nighttime symptoms on HRQL are lacking at the moment and constitute another area for research.

\section{How to detect and measure symptom variability}

As subjective information given by the patients, symptom perception must be measured by the use of validated questionnaires. In the last few years, several questionnaires have been developed to assess symptoms in COPD. The first clinical questionnaire developed to assess symptoms in COPD was the Clinical COPD Questionnaire (CCQ). ${ }^{24}$ Others have followed, aiming to evaluate different symptoms or aspects of the disease, including the Leicester Cough Questionnaire, which assesses the effects of chronic cough on health status ${ }^{25}$ the Manchester Respiratory Activities Of Daily Living questionnaire, which assesses respiratory disability in elderly outpatients with COPD $;{ }^{26}$ and the Cough And Sputum Assessment Questionnaire for the assessment of chronic bronchitis. ${ }^{27}$ However, none of these have aimed to evaluate the variability of the measured symptoms at different times of the day.

The Capacity of Daily Living during the Morning (CDLM) questionnaire, developed by Partridge et al, is the first tool specifically designed to assess the impact of symptoms on the activities during the first hours of the morning. ${ }^{19}$ The CDLM questionnaire consists of six questions that ask the patient if he/she is able to perform six common daily morning activities and evaluate the degree of difficulty they normally experience. Each item in the CDLM questionnaire is scored on a scale ranging from 0 (the activity cannot be carried out by the patient) to 5 (the activity is not difficult at all to carry out). The minimal clinically important difference (MCID) is 0.20 .

Although no specific questionnaire for COPD nighttime symptoms is available, there are some valuable initiatives. The COPD and Asthma Sleep Impact Scale (CASIS) questionnaire was developed as a self-report measure to assess the impact of chronic respiratory diseases (CODP and asthma) on sleep quality. ${ }^{28}$ This questionnaire was based on COPD and asthma patient interviews, and it is composed of seven items. CASIS is significantly correlated with the St George Respiratory Questionnaire $^{28}$ and differs with the disease severity and exacerbations. The usefulness of this questionnaire was assessed in 408 COPD patients, those patients with several symptoms and clinical features had higher scores. These symptoms were chronic cough, expectoration, level of dyspnea, number of exacerbations, activities of daily living, depression and anxiety syptoms and number of treatments indicated. ${ }^{29}$ Additionally, in the Aclidinium in Chronic Obstructive Respiratory Disease I (ACCORD I) study, COPD symptoms (in early morning and at night) and rescue medication use were assessed using the Nighttime Symptoms Questionnaire. ${ }^{30}$ Unfortunately, information on the validation of this questionnaire is not available to date.

Irrespective of the questionnaires used, in the actual era towards personalized medicine, ${ }^{31}$ it is important to call all respiratory professionals to pay attention to the distribution of symptoms during the day, since this may help us identify different patient types and to establish a proper treatment strategy to mitigate the impact of these symptoms on HRQL. In this regard, health care providers managing COPD patients should include questions related to morning and nighttime symptoms in their clinical interviews as a way to detect these variations. Even a simple question, such as what time of the day the patient feels more symptomatic or how the patient deals with the first hours of the day, may be extremely informative.

\section{How to manage symptom variability}

Despite the described evidence of variation in perceived symptoms during the day, this appears to be hardly considered 
in actual treatment plans. ${ }^{32}$ In the study by Kessler et al, ${ }^{11} 51.3 \%$ of patients declared they did not alter their treatment despite symptom worsening during the day, and only $35.7 \%$ reported an increased use of their rescue inhaler. Additionally, $38.7 \%$ of patients declared they did not change medication even when the chest symptoms worsened over several days. So there is a need to provide health care professionals with treatment strategies able to lessen the impact of this variation.

The impact of treatment on morning symptoms was evaluated in a recent trial comparing the effect of salmeterol/ fluticasone versus budesonide/formoterol on lung function, symptoms, and activities in the early morning. ${ }^{23}$ This was a randomized, double-blind, crossover study including 442 patients with COPD with mean $\mathrm{FEV}_{1} 36.1 \%$ (13\%$51 \%$ ). Although treatment with budesonide/formoterol or salmeterol/fluticasone was effective in the patients with COPD, budesonide/formoterol had a more rapid onset of effect compared with salmeterol/fluticasone, which resulted in greater improvements in the patient's ability to perform morning activities close to the MCID of the questionnaire. This difference was attributed to the more rapid onset of action of formoterol versus salmeterol, despite the lower inhaled corticosteroid dose in the budesonide regimen.

Although this hypothesis remains to be validated in future long-term clinical trials comparing fast-onset versus slowonset inhaled drugs in COPD, this finding opened an interesting new debate on the use of rapid-acting bronchodilators in the treatment of COPD. Until recently, it was thought that the speed of action was not an important issue in the treatment of patients with a chronic disease such as COPD. Considering that the patient was under the influence of bronchodilators 24 hours a day, it seemed not to be important that the next morning dose of the same drug had a faster or slower onset of action. However, these studies suggest that the faster the drug acts, the faster the improvement experienced by the patient, and this may have an impact on morning symptoms. Interestingly, the CLIMB study, a 12-week study evaluating the efficacy of adding tiotropium to formoterol/budesonide in 660 subjects with COPD, ${ }^{33}$ found that budesonide/formoterol plus tiotropium provided rapid and significant improvements in morning symptoms at 5 and 15 minutes compared with tiotropium alone, as measured by the CDLM questionnaire. Although this hypothesis should be validated in future longterm clinical trials comparing the effect of fast onset versus slow onset inhaled drugs on HRQL and the activities of the daily living, it may lead to new thinking on long-term bronchodilator therapy in COPD.

At the present time, the two available LABAs for the treatment of COPD that have a fast onset of action are formoterol, taken twice daily, and the recently marketed, once-daily indacaterol. Indacaterol is an ultra-LABA with a rapid onset of action and 24-hour duration ${ }^{34}$ that has high efficacy and safety in the treatment of COPD, either as stand-alone therapy ${ }^{35}$ or as an add-on therapy. ${ }^{36}$ There are data indicating that newer long-acting muscarinic antagonists (LAMA) also have a faster onset of action. In the GLOW2 trial comparing the efficacy and safety of once-daily glycopyrronium (NVA237) versus placebo and open-label tiotropium, glycopyrronium provided faster bronchodilation with significantly higher $\mathrm{FEV}_{1}$ at all time points, from 5 minutes to 4 hours postdose, compared with the placebo and with tiotropium. ${ }^{37}$ Interestingly, a potential mechanism underlying this faster onset of action of glycopyrronium versus tiotropium, beyond the dissociation from the $\mathrm{M}_{3}$ receptor, has been recently hypothesized. ${ }^{38}$ In preclinical studies, aclidinium showed a faster onset of action than tiotropium in isolated guinea pig trachea, ${ }^{39}$ but this effect was not reproduced in a clinical setting where both drugs had similar performance. ${ }^{40}$ The extended use of all these new drugs - the clinical experience and the coming new trials - will shed further light on the effect of the rapid onset of action and their impact on clinically meaningful patient-centered outcomes.

\section{Conclusion}

The prior concept of COPD, as a constant and inexorably progressing disease in which symptoms increase with deteriorating lung function, may not apply to a particular patient. Symptoms are variable with time, changing with the seasons, within a week, and even over the same day. Previous studies show that many patients experience an increase of disease-related symptoms during the morning and/or during the nighttime that impacts on daily activities and HRQL. In this regard, there is new evidence showing that fast-onset bronchodilators (LABAs and LAMAs) may have an additional role in the control of these morning symptoms and improvement in HRQL. Future trials are needed to assess the impact of this new clinical outcome in the management of COPD. Health care professionals caring for COPD patients should consider this variation in the perception of symptoms during their clinical interview, as a potential new target in the long-term treatment plan, which will be further clarified in the future.

\section{Acknowledgment}

The authors are thankful to Novartis for editorial support of the present manuscript. 


\section{Disclosure}

JLLC has received honoraria for lecturing, scientific advice, participation in clinical studies or writing for publications for (alphabetial order): Almirall, AstraZeneca, Bayer, Boehringer Ingelheim, Cantabria Pharma, Chiesi, Esteve, Faes, Ferrer, GlaxoSmithKline, Menarini, MSD, Novartis, Pfizer and Takeda (Nycomed). CC and EQG report no conflicts of interest in this work.

\section{References}

1. Viejo-Bañuelos JL, Pueyo-Bastida A, Fueyo-Rodriguez A. Characteristics of outpatients with COPD in daily practice: The E4 Spanish project. Respir Med. 2006;100(12):2137-2143.

2. Soriano JB, Calle M, Montemayor T, Alvarez-Sala JL, Ruiz-Manzano J, Miravitlles M. The general public's knowledge of chronic obstructive pulmonary disease and its determinants: current situation and recent changes. Arch Bronconeumol. 2012;48(9):308-315. Spanish.

3. Fletcher C, Peto R. The natural history of chronic airflow obstruction. Br Med J. 1977;1(6077):1645-1648.

4. Vestbo J, Edwards LD, Scanlon PD, et al. Changes in forced expiratory volume in 1 second over time in COPD. New Engl J Med. 2011; 365(13):1184-1192.

5. Kohansal R, Martinez-Camblor P, Agustí A, Buist AS, Mannino DM, Soriano JB. The natural history of chronic airflow obstruction revisited: an analysis of the Framingham offspring cohort. Am J Respir Crit Care Med. 2009;180(1):3-10.

6. Casanova C, de Torres JP, Aguirre-Jaíme A, et al. The progression of chronic obstructive pulmonary disease is heterogeneous: the experience of the BODE cohort. Am J Respir Crit Care Med. 2011; 184(9):1015-1021.

7. Agusti A, Calverley PM, Celli B, et al; Evaluation of COPD Longitudinally to Identify Predictive Surrogate Endpoints (ECLIPSE) Investigators. Characterisation of COPD heterogeneity in the ECLIPSE cohort. Respir Res. 2010;11:122.

8. López-Campos JL. Treatment strategies in chronic obstructive pulmonary disease: a proposal for standardization. Arch Bronconeumol. 2010;46(12):617-620. Spanish.

9. Joshi M, Joshi A, Bartter T. Symptom burden in chronic obstructive pulmonary disease and cancer. Curr Opin Pulm Med. 2012; 18(2):97-103.

10. Partridge MR, Karlsson N, Small IR. Patient insight into the impact of chronic obstructive pulmonary disease in the morning: an internet survey. Curr Med Res Opin. 2009;25(8):2043-2048.

11. Kessler R, Partridge MR, Miravitlles M, et al. Symptom variability in patients with severe COPD: a pan-European cross-sectional study. Eur Respir J. 2011;37(2):264-272.

12. Jenkins CR, Celli B, Anderson JA, et al. Seasonality and determinants of moderate and severe COPD exacerbations in the TORCH study. Eur Respir J. 2012;39(1):38-45.

13. Jones PW, Brusselle G, Dal Negro RW, et al. Patient-centred assessment of COPD in primary care: experience from a cross-sectional study of health-related quality of life in Europe. Prim Care Respir J. 2012; 21(3):329-336.

14. Espinosa de los Monteros MJ, Peña C, Soto Hurtado EJ, Jareño J, Miravitlles M. Variability of respiratory symptoms in severe COPD. Arch Bronconeumol. 2012;48(1):3-7. English, Spanish.

15. Kim YJ, Lee BK, Jung CY, et al. Patient's perception of symptoms related to morning activity in chronic obstructive pulmonary disease: the SYMBOL study. Korean J Intern Med. 2012; 27(4): 426-435.

16. Schnell K, Weiss CO, Lee T, et al. The prevalence of clinicallyrelevant comorbid conditions in patients with physician-diagnosed COPD: a cross-sectional study using data from NHANES 1999-2008. BMC Pulm Med. 2012;12:26.
17. Donaldson GC, Goldring JJ, Wedzicha JA. Influence of season on exacerbation characteristics in patients with COPD. Chest. 2012;141(1): 94-100.

18. Barba R, Zapatero A, Losa JE, et al. The impact of weekends on outcome for acute exacerbations of COPD. Eur Respir J. 2012; 39(1):46-50

19. Partridge MR, Miravitlles M, Ståhl E, Karlsson N, Svensson K, Welte T. Development and validation of the Capacity of Daily Living during the Morning questionnaire and the Global Chest Symptoms Questionnaire in COPD. Eur Respir J. 2010;36(1):96-104.

20. Agusti A, Hedner J, Marin JM, Barbé F, Cazzola M, Rennard S. Night-time symptoms: a forgotten dimension of COPD. Eur Respir Rev. 2011;20(121):183-194.

21. Wouters EF, Postma DS, Fokkens B, et al; COSMIC (COPD and Seretide: a Multi-Center Intervention and Characterization) Study Group. Withdrawal of fluticasone propionate from combined salmeterol/fluticasone treatment in patients with COPD causes immediate and sustained disease deterioration: a randomised controlled trial. Thorax. 2005;60(6):480-487.

22. van den Berge $M$, Hop WC, van der Molen $\mathrm{T}$, et al; COSMIC (COPD and Seretide: a Multi-Center Intervention and Characterization) Study Group. Prediction and course of symptoms and lung function around an exacerbation in chronic obstructive pulmonary disease. Respir Res. 2012;13:44.

23. Partridge MR, Schuermann W, Beckman O, Persson T, Polanowski T. Effect on lung function and morning activities of budesonide/formoterol versus salmeterol/fluticasone in patients with COPD. Ther Adv Respir Dis. 2009;3(4):1-11.

24. van der Molen T, Willemse BW, Schokker S, ten Hacken NH, Postma DS, Juniper EF. Development, validity and responsiveness of the Clinical COPD Questionnaire. Health Qual Life Outcomes. 2003;1:13

25. Berkhof FF, Boom LN, ten Hertog NE, Uil SM, Kerstjens HA, van den Berg JW. The validity and precision of the Leicester Cough Questionnaire in COPD patients with chronic cough. Health Qual Life Outcomes. 2012;10:4.

26. Yohannes AM, Roomi J, Winn S, Connolly MJ. The Manchester Respiratory Activities of Daily Living questionnaire: development, reliability, validity, and responsiveness to pulmonary rehabilitation. J Am Geriatr Soc. 2000;48(11):1496-1500.

27. Monz BU, Sachs P, McDonald J, Crawford B, Nivens MC, Tetzlaff K. Responsiveness of the cough and sputum assessment questionnaire in exacerbations of COPD and chronic bronchitis. Respir Med. 2010;104(4):534-541.

28. Pokrzywinski RF, Meads DM, McKenna SP, Glendenning GA, Revicki DA. Development and psychometric assessment of the COPD and Asthma Sleep Impact Scale (CASIS). Health Qual Life Outcomes. 2009;7:98.

29. Miravitlles M, Iriberri M, Barrueco M, Lleonart M, Villarrubia E, Galera J. Usefulness of the LCOPD, CAFS and CASIS Scales in understanding the impact of COPD on patients. Respiration. Epub October 2, 2012.

30. Kerwin EM, D’Urzo AD, Gelb AF, Lakkis H, Garcia Gil E, Caracta CF; ACCORD I study investigators. Efficacy and safety of a 12-week treatment with twice-daily aclidinium bromide in COPD patients (ACCORD COPD I). COPD. 2012;9(2):90-101.

31. Sobradillo P, Pozo F, Agustí A. P4 medicine: the future around the corner. Arch Bronconeumol. 2011;47(1):35-40.

32. Rodríguez-Roisin R, Agustí A. The GOLD initiative 2011: a change of paradigm? Arch Bronconeumol. 2012;48(8):286-289. English, Spanish.

33. Welte T, Miravitlles M, Hernandez P, et al. Efficacy and tolerability of budesonide/formoterol added to tiotropium in patients with chronic obstructive pulmonary disease. Am J Respir Cit Care Med. 2009;180(8): 741-750.

34. Kato M, Makita H, Uemura K, et al. Bronchodilator efficacy of single doses of indacaterol in Japanese patients with COPD: A randomised, double-blind, placebo-controlled trial. Allergol Int. 2010;59(3):285-293. 
35. Decramer M, Rossi A, Lawrence D, McBryan D. Indacaterol therapy in patients with COPD not receiving other maintenance treatment. Respir Med. 2012;106(12):1706-1714.

36. Mroz RM, Minarowski L, Chyczewska E. Indacaterol add-on therapy improves lung function, exercise capacity and life quality of COPD patients. Adv Exp Med Biol. 2013;756:23-28.

37. Kerwin E, Hébert J, Gallagher N, et al. Efficacy and safety of NVA237 versus placebo and tiotropium in patients with COPD: the GLOW2 study. Eur Respir J. 2012;40(5):1106-1114.

38. Sykes DA, Dowling MR, Leighton-Davies J, et al. The Influence of receptor kinetics on the onset and duration of action and the therapeutic index of NVA237 and tiotropium. J Pharmacol Exp Ther. 2012; 343(2):520-528.
39. Gavaldà A, Miralpeix M, Ramos I, et al. Characterization of aclidinium bromide, a novel inhaled muscarinic antagonist, with long duration of action and a favorable pharmacological profile. J Pharmacol Exp Ther. 2009;331(2):740-751.

40. Vestbo J, Vogelmeier C, Creemers J, Falques M, Ribera A, Gil EG. Onset of effect of aclidinium, a novel, long-acting muscarinic antagonist, in patients with COPD. COPD. 2010;7(5):331-336.

\section{Publish your work in this journal}

The International Journal of COPD is an international, peer-reviewed journal of therapeutics and pharmacology focusing on concise rapid reporting of clinical studies and reviews in COPD. Special focus is given to the pathophysiological processes underlying the disease, intervention programs, patient focused education, and self management protocols.

\section{Dovepress}

This journal is indexed on PubMed Central, MedLine and CAS. The manuscript management system is completely online and includes a very quick and fair peer-review system, which is all easy to use. Visit $\mathrm{http} / / / \mathrm{www}$.dovepress.com/testimonials.php to read real quotes from published authors.

Submit your manuscript here: http://www.dovepress.com/international-journal-of-copd-journal 